\title{
The relationship between speech perceptual discrimination and speech production in Parkinson's Disease
}

Article

Accepted Version

Mollaei, F. ORCID: https://orcid.org/0000-0003-2916-9750, Shiller, D. M., Baum, S. R. and Gracco, V. L. (2019) The relationship between speech perceptual discrimination and speech production in Parkinson's Disease. Journal of Speech Language and Hearing Research, 62 (12). pp. 4256-4268. ISSN 1558-9102 doi: https://doi.org/10.1044/2019_JSLHR-S18-0425 Available at https://centaur.reading.ac.uk/97894/

It is advisable to refer to the publisher's version if you intend to cite from the work. See Guidance on citing.

To link to this article DOI: http://dx.doi.org/10.1044/2019_JSLHR-S-18-0425

Publisher: ASHA

All outputs in CentAUR are protected by Intellectual Property Rights law, including copyright law. Copyright and IPR is retained by the creators or other copyright holders. Terms and conditions for use of this material are defined in the End User Agreement. 


\section{CentAUR}

Central Archive at the University of Reading

Reading's research outputs online 
The relationship between speech perceptual discrimination and speech production in Parkinson's disease

Title: The relationship between speech perceptual discrimination and speech production in Parkinson's disease

Authors:

Fatemeh Mollaei, PhD ${ }^{\text {a,b }}$, Douglas M. Shiller, $\mathrm{PhD}{ }^{\text {a,c }}$, Shari R. Baum, $\mathrm{PhD}{ }^{\text {a,b }}$, Vincent L. Gracco, $\mathrm{PhD}$ a,b,d

${ }^{\text {a }}$ Centre for Research on Brain, Language and Music, Montreal, Canada

${ }^{\mathrm{b}}$ School of Communication Sciences and Disorders, McGill University, Montreal, Canada

${ }^{c}$ School of Speech-Language Pathology and Audiology, Université de Montréal, Montreal, Canada

d Haskins Laboratories, New Haven, CT, USA

\section{Corresponding author:}

Fatemeh Mollaei

Centre for Research on Brain, Language and Music

McGill University

3640 rue de la Montagne

Montreal, Quebec H3G 2A8, Canada

Phone: +1-514-806-7051

E-mail: fatemeh.mollaei@mail.mcgill.ca

Total number of words in manuscript: 7,826

Words in abstract: 234

Number of tables and figures: 6

Running Title: Perceptual speech deficits in Parkinson's disease

Key Words: Perceptual processing, sensorimotor control, Parkinson's disease, vocal pitch, formant frequencies

Funding statement: This work was supported by grants from the Natural Sciences and Engineering Research Council of Canada (NSERC) awarded to Dr. Baum and Dr. Gracco.

Financial disclosure of all authors/conflicts of interest: None. 
The relationship between speech perceptual discrimination and speech production in Parkinson's disease

\section{Abstract}

Purpose: We recently demonstrated that individuals with Parkinson's disease (PD) respond differentially to specific altered auditory feedback parameters during speech production. Participants with PD respond more robustly to pitch and less robustly to formant manipulations compared to control participants. In this study, we investigated whether differences in perceptual processing may in part underlie these compensatory differences in speech production.

Methods: Pitch and formant feedback manipulations were presented under two conditions: production and listening. In the production condition, fifteen participants with PD and fifteen age- and gender-matched healthy control participants judged whether their own speech output was manipulated in real-time. During the listening task, participants judged whether paired tokens of their previously recorded speech samples were the same or different.

Results: Under listening, first formant manipulation discrimination was significantly reduced for the PD group compared to the control group. There was a trend toward better discrimination of pitch in the PD group, but the group difference was not significant. Under the production condition, the ability of participants with PD to identify pitch manipulations was greater than that of the controls.

Conclusions: The findings suggest perceptual processing differences associated with acoustic parameters of fundamental frequency and first formant perturbations in PD. These findings extend our previous results, indicating that different patterns of compensation to pitch and first formant shifts may reflect a combination of sensory and motor mechanisms that are differentially influenced by basal ganglia dysfunction. 
The relationship between speech perceptual discrimination and speech production in Parkinson's disease

\section{Introduction}

Approximately $90 \%$ of individuals with PD experience speech and auditory processing disorders (Duffy, 2013). The speech disorder of PD, hypokinetic dysarthria, consists of disturbances in prosody, phonation, and articulation (Duffy, 2013). Phonatory and laryngeal deficits are among the most common speech disturbances of hypokinetic dysarthria, and while reduced loudness is the most prominent laryngeal symptom, reduced fundamental frequency $\left(f_{\mathrm{o}}\right)$ and monopitch are also among the cardinal disturbances of speech (Skodda, Rinsche, \& Schlegel, 2009; Skodda, Visser, \& Schlegel, 2011a, 2011b; Duffy, 2013). The articulation deficits in PD include a restriction of vowel formant frequencies and imprecise consonants (Duffy, 2013; Roy, Nissen, \& Dromey, 2009; Skodda, Groenheit, \& Schlegel, 2012). It has been argued that speech disorders in PD are not solely motor in nature, but also have sensory and perceptual components (Arnold, Gehrig, Gisport, Seifried, \& Kell, 2014; Chen et al., 2013; Liu, Wang, Metman, \& Larson, 2012; Mollaei, Shiller, Baum, \& Gracco, 2016). For example, in a functional MRI study, Arnold et al. (2014) found reduced activation associated with monitoring of auditory feedback. They suggested that this reduced monitoring might underlie inaccurate updating of motor representations that manifest in dysarthria.

In addition, it has been found that individuals with PD are not able to correctly scale their speech output in the absence of any external cue, although they are able to do so under explicit instructions (Clark, Adams, Dykstra, Moodie, \& Jog, 2014; Fox \& Ramig, 1997; Ho, Bradshaw, \& Iansek, 2000; Kwan \& Whitehill, 2011). These internal self-correction deficits suggest that difficulties in the monitoring of speech production might be related to the way auditory information is being perceived. This deficit would be consistent with reduced levels of attention during speech perception in PD (Brown \& Marsden, 1988). However, little is known about 
The relationship between speech perceptual discrimination and speech production in Parkinson's disease

sensory processing related to speech production in PD. To investigate the sensory contributions to speech production, real-time perturbations of auditory feedback can be used to evaluate errorbased corrections. This requires processing the error and correcting for it. The perturbations can be predictable, known as sensorimotor adaptation, or unpredictable, used to assess the online sensorimotor control process, known as sensorimotor compensation. Healthy speakers correct their speech output by compensating for perceived auditory feedback alterations (Purcell \& Munhall, 2006; Tourville, Reilly, \& Guenther, 2008; Larson, Burnett, Kiran, \& Hain, 2000). In PD, individuals exhibit differences in their ability to correct for auditory feedback errors, as shown using both compensation and adaptation paradigms, reflecting a deficit in integrating the error signal with a change in motor output (Abur et al., 2018a; Chen et al., 2013; Huang et al., 2016; Liu et al., 2012; Mollaei et al., 2016; Mollaei, Shiller, \& Gracco, 2013). However, not all studies have found different patterns in PD relative to controls. For example, Kiran and Larson (2001) did not find any differences in the magnitude of the response using a pitch-altered auditory feedback compensation paradigm in individuals with PD; nonetheless, they did find differences in temporal aspects of the response. It should also be noted that they tested individuals with PD on medication (within 2 hours of medication intake) while Liu et al. (2012), Chen et al. (2013), Huang et al. (2016), and Mollaei et al. (2016) tested individuals off medication for at least 12 hours. These differences may account for the inconsistent patterns of results.

Speech production involves multiple subsystems, most notably laryngeal and articulatory, and while these two subsystems function together, they also can be tuned and modulated independently (Liu, Auger, \& Larson, 2010; MacDonald, Purcell, \& Munhall, 2011). These two subsystems have been shown to be differentially susceptible to real-time manipulations of 
The relationship between speech perceptual discrimination and speech production in Parkinson's disease

sensory feedback (Larson et al., 2000; Purcell \& Munhall, 2006). $f_{\mathrm{o}}$ and loudness are suprasegmental laryngeal parameters in English and appear to be more sensitive to moment-tomoment changes in auditory feedback (Larson et al., 2000), and they tend to change rapidly with a change in hearing status (Perkell et al., 1997; Svirsky et al., 1992), suggesting that they are controlled independently or at least differentially from supralaryngeal articulatory parameters, which exhibit different patterns. Formant frequencies, including first formant frequency $\left(F_{1}\right)$, are mainly a reflection of movement of the tongue shaping the vocal tract and contribute to the quality of a given vowel. $F_{1}$ has been shown to be less sensitive than $f_{\mathrm{o}}$ to rapid changes in auditory feedback, with compensatory changes occurring more slowly (Cowie \& DouglasCowie, 1992; Perkell et al., 2000). In addition, the laryngeal disturbances associated with PD seem to have a different timeline compared to articulatory deficits (Ho, Iansek, Marigliani, Bradshaw, \& Gates, 1999; Skodda, Gronheit, \& Schlegel, 2012). Based on these findings and the independence of articulatory and laryngeal features of speech, we hypothesized a different pattern of processing of $f_{\mathrm{o}}$ and $F_{1}$ in $\mathrm{PD}$.

Different acoustic properties of speech encode information associated with these different speech motor subsystems. A number of studies have found increased $f_{\mathrm{o}}$ compensation for pitch shifts in PD (Chen et al., 2013; Huang et al., 2016; Liu et al., 2012; Mollaei et al., 2016). Using EEG, Huang et al. (2016) reported larger P2 responses associated with increased compensation of pitch in individuals with PD compared to control participants during vocalization. This larger response was left-lateralized in the superior and inferior frontal gyrus, premotor cortex, inferior parietal lobule, and superior temporal gyrus. Presumably, the enhanced EEG response is consistent with increased sensitivity to $f_{\mathrm{o}}$ changes and/or increased weighting of auditory information for pitch control. In our previous study (Mollaei et al., 2016), we observed that 
The relationship between speech perceptual discrimination and speech production in Parkinson's disease

individuals with PD compensate differently for changes in $f_{\mathrm{o}}$ compared to their response to changes in $F_{1}$ during vowel production. Interestingly, participants with PD responded more robustly than controls to pitch perturbations (cf. Chen et al., 2013; Liu et al., 2012), while they responded less robustly to $F_{1}$ perturbations. Based on our previous work, it appears that PD may affect the underlying sensorimotor mechanisms for laryngeal and articulatory subsystems differentially. The source (sensory, motor, sensorimotor) of this differential effect is the main focus of this study.

There are a few studies that have investigated speech perception in PD during passive listening tasks (Abur, Lupiani, Hickox, Shinn-Cunningham, \& Stepp, 2018b; Clark et al., 2014; De Keyser et al., 2016; Dromey \& Adams, 2000; Huang et al., 2016; Troche, Troche, Berkowitz, Grossman, \& Reilly, 2012). Troche et al. (2012) reported reduced discrimination of frequency and amplitude of pure tones, and Clark et al. (2014) found decreased sensitivity to differences in speech loudness during listening using a magnitude estimation task. However, other studies reported no speech perception deficits during listening in PD (Abur et al., 2018b; Dromey \& Adams, 2000; Huang et al., 2016). For example, Abur et al. (2018b) found no group differences in loudness perception for pure tones using a rating scale. These contradictory findings might be explained by the nature of the auditory information under investigation (i.e., pitch and loudness of speech sounds vs. pure tones) as well as differences in the tasks used (i.e., discrimination vs. magnitude estimation).

While the above-mentioned studies investigated perceptual deficits in $\mathrm{PD}$, the focus has primarily been on loudness (Abur et al., 2018b; Brajot, Shiller, \& Gracco, 2016; Clark et al., 2014; De Keyser et al., 2016; Ho et al., 2000), lexical (discourse) level attributes (McNamara, Obler, Au, Durso, \& Albert, 1992), and auditory perception of pure tone differences (Abur et al., 
The relationship between speech perceptual discrimination and speech production in Parkinson's disease

2018b; Troche et al., 2012) rather than at a level of acoustic attributes associated with laryngeal and articulatory control. Here we focus on two aspects of perceptual processing for both laryngeal and articulatory changes: the ability to monitor feedback changes during production and the more general ability to detect differences in pitch and formant manipulations during listening. These two perceptual processes are different in that self-monitoring after production requires comparison of feedback with an internal representation of the intended production (Niziolek, Nagarajan, \& Houde, 2013), while auditory discrimination is an ability to recognize differences between sounds during listening that may not engage internal representations of the sound. It has been found that cortical and subcortical structures involved in self-monitoring during production and listening are distinct (Behroozmand et al., 2015).

In the present study, we investigated PD participants' ability to self-monitor and detect changes in $f_{\mathrm{o}}$ and $F_{1}$ parameters during speech production and perception and compared these abilities to their sensorimotor compensatory responses. In keeping with the finding of increased pitch compensation (Chen et al., 2013; Huang et al., 2016; Liu et al., 2012; Mollaei et al., 2016), we hypothesized that participants with PD would be better able to detect pitch errors compared to control participants during production. Similarly, in line with the finding of reduced compensation to $F_{1}$ alterations found in our previous studies (Mollaei et al., 2013, 2016), we predicted that participants with PD would show a reduced ability to detect $F_{1}$ errors during production. During listening, we hypothesized a perceptual processing deficit in PD that would result in differences in the ability to detect errors in $f_{\mathrm{o}}$ and $F_{1}$ between the two groups. Such an outcome would indicate a deficit in PD that is separable from the motor dysfunction. Teasing apart sensorimotor versus perceptual deficits in PD is an important first step in understanding the mechanisms involved in speech monitoring in PD. 
The relationship between speech perceptual discrimination and speech production in Parkinson's disease

\section{Methods}

\subsection{Ethics statement}

This study was approved by the McGill Faculty of Medicine Institutional Review Board, in accordance with principles expressed in the Declaration of Helsinki. Informed written consent was obtained from participants prior to their involvement in the project.

\subsection{Participants}

Fifteen participants with PD (6 female, 9 male; mean age: 65.87 years) and fifteen age- and gender-matched control participants (6 female, 9 male; mean age: 61.58 years) were recruited for this study. These are the same participants who participated in our previous study (Mollaei et al., 2016). The severity of PD motor symptoms, which we assessed using the Movement Disorder Society Unified Parkinson's Disease Rating Scale (MDS-UPDRS; Part III Motor Examination), ranged from mild (a score of 13 ) to moderate (a score of 48; mean \pm standard deviation [SD] score: $24.79 \pm 9.19)$ (Fahn, Elton, \& Committee, 1987). The MDS-UPDRS administrator received in-house training at the Speech Motor Control Laboratory at McGill University. Cognitive functioning was assessed using the Montreal Cognitive Assessment (MoCA, Nasreddine et al., 2005) and was in the normal range for all participants with PD and healthy controls (scores $>26$ ). All participants with PD were taking L-dopa but were tested off medication (12 hours). Two participants with PD had a history of speech therapy that focused on enhancing loudness and intelligibility.

Participants underwent an audiometric screening and were found to have binaural pure tone hearing thresholds of $40 \mathrm{~dB} \mathrm{HL}$ or less at 250, 500, 1000, 2000 and $4000 \mathrm{~Hz}$. We included 
The relationship between speech perceptual discrimination and speech production in Parkinson's disease

participants with mild hearing loss because the basis of our comparison was between healthy age- and gender-matched control participants and participants with PD, and both groups showed mild hearing loss (as in our previous study, Mollaei et al., 2016). All participants were native speakers of North-American English. None used hearing aids or had any other neurological disease or surgical intervention, such as deep brain stimulation, pallidotomy, or thalamotomy.

A standard speech reading assessment (Rainbow Passage; Fairbanks, 1960) was used to rate 43 perceptual characteristics related to phonatory (e.g., loudness and pitch), articulatory, resonatory, prosodic, and respiratory characteristics of speech. A licensed Speech-Language Pathologist listened to the speech samples of each participant and rated the speech on each characteristic using a 7-point scale (1 representing normal speech and 7 representing severe speech deviation) as described in the original reports by Darley, Aronson and Brown (1969) and 43 perceptual dimensions classification system expanded by Duffy (2013). We used this method to characterize the subtypes of dysarthria as well as the severity of speech disturbances in each individual with PD, and to correlate the severity of dysarthria with error detection responses. The hypokinetic dysarthria subtype was found in participants with PD, with severity ratings of moderate (2 participants), mild-to-moderate (4 participants), mild (6 participants), and within normal range (3 participants). A second rater evaluated the same sample of speech. Inter-rater reliability was assessed using intra-class correlation (ICC) in order to assess consistency in the ratings of speech perceptual characteristics in individuals with PD. The resulting ICC was in the excellent range, ICC $=0.90$ (Cicchetti, 1994), indicating that the raters had a high degree of agreement. The MDS-UPDRS and perceptual scores (presented in Table 1) were used to assess any relationship between severity of motor and speech symptoms with their speech perceptual ability. 
The relationship between speech perceptual discrimination and speech production in Parkinson's disease

\subsection{Stimuli and experimental design}

The study consisted of two separate experiments, each involving the manipulation of $f_{\mathrm{o}}$ and $F_{1}$. In the first experiment (Production $-f_{\mathrm{o}}$ and Production $-F_{1}$ ), participants repeatedly produced the target vowel $[\varepsilon]$ in the context of word "head" ([hed]) and, after each production trial, they had to indicate whether the sound of their own voice (presented in near-real time over headphones) was the same or different from what they had produced. For the $f_{\mathrm{o}}$ manipulation condition, 180 trials were carried out with half of the trials involving a manipulation of feedback (30 trials at each of three magnitudes: small, medium and large - see below for details). For the $F_{1}$ manipulation condition, 120 trials were produced with half involving a feedback manipulation (30 trials at each of two magnitudes: small and large - see below for details). Altogether, $90 f_{\mathrm{o}}$ perturbation trials, and $60 F_{1}$ perturbation trials were acquired, yielding 150 perturbation trials in total for each participant. In order to assess any potential compensation associated with the $f_{\mathrm{o}}$ or $F_{1}$ manipulations, participants were instructed to sustain the vocalic portion of the utterance for 2.5 seconds. The choice of the stimulus and its duration was consistent with our previous study (Mollaei et al., 2016), and while it may be somewhat different from typical word production, our main aim was the comparison between the two participant groups. In the production experiment (Experiment 1), the perturbed trials were quasi-randomized with the constraint that two shifted trials of the same magnitude would not occur consecutively.

In the second experiment (Listening $-f_{\mathrm{o}}$ and Listening $-F_{1}$ ), participants listened to pairs of pre-recorded samples of their own speech recorded during the first experiment and completed an auditory discrimination task (AX paradigm, with a "same" or "different" judgment following each presented pair of stimuli) using the same manipulations and magnitudes of feedback error induced in $f_{\mathrm{o}}$ and $F_{1}$. As in Experiment 1, the second experiment included 300 trials in total, 150 
The relationship between speech perceptual discrimination and speech production in Parkinson's disease

of which involved a perturbation, with 90 "different" trials involving $f_{\mathrm{o}}$ perturbations, 60

“different" trials involving $F_{1}$ perturbations, and 150 "same" trials, hence counterbalancing the

number of same versus different trials. Participants were instructed to make same/different

judgments as quickly and accurately as possible for each presented pair of words. We chose this

design because it is suitable for exploring perceptual accuracy based on the changes that arise during speech production (Macmillan \& Creelman, 2005). The order of stimulus presentation and alteration magnitude was randomized. At the level of an individual trial (each consisting of a pair of words), the inter-stimulus interval (ISI) was $400 \mathrm{~ms}$ in order to reduce the auditory memory load of task in participants with PD and older healthy controls (Troche et al., 2012). The duration of the stimuli in the listening task was the same as the Production task and equal to 2.5 seconds. The loudness of each stimulus in the listening task was adjusted based on each participant's production, amplified to a volume of $70 \mathrm{~dB}$ SPL to be consistent with the Production task. Each pair of stimuli was normalized to ensure equal loudness and duration. For both experiments, participants were first tested under one shift condition $\left(f_{\mathrm{o}}\right.$ or $F_{1}$ shift) followed by a short rest period (approximately 10 minutes) and then tested under the second condition. The order of manipulation conditions was counterbalanced among participants. $f_{\mathrm{o}}$ was manipulated in an increasing direction, because it has been previously shown that this perturbation elicits a significantly larger response magnitude compared with a reduction in $f_{\mathrm{o}}$ (Chen et al., 2013). The manipulation of $F_{1}$ was also in an increasing direction (in the direction of $[\varepsilon]$ to $[æ]$ in the vowel space), in line with our previous study using a vowel formant adaptation paradigm (Mollaei et al., 2013). 
The relationship between speech perceptual discrimination and speech production in Parkinson's disease

\subsection{Manipulation of auditory feedback}

The participants' voices were transduced using a head-mounted microphone (C520, AKG, Germany) that was located $20 \mathrm{~cm}$ from participants' mouths. The microphone signal was digitized at 22,050 Hz/16-bit (Fast-Track Pro, M-Audio, Irwindale, CA), and was recorded on a PC using MATLAB (The Mathworks, Inc., Natick, MA). In parallel with the recording, the acoustical signal was processed in near-real-time using a VoiceOne vocal processor (TC Helicon) and played back to participants through circumaural headphones (880 Pro, Bayerdynamic, Germany).

For the shifting of $F_{1}$, the speech processing involved pre-amplification of the microphone signal, and then splitting the line-level signal into two channels. In one channel, the VoiceOne was used to shift all formant frequencies by $15 \%$ or $30 \%$ (depending on the condition), after which the signal was analog low-pass filtered (Model 751A, Rockland) in order to isolate the $F_{1}$ (using a cut-off frequency of 1100 for males, and 1350 for females). A second channel consisting of an unaltered speech signal (no formant shift) was high-pass filtered using the same cut-off frequency, and then mixed with the low-passed formant shifted signal, thereby creating a full-spectrum speech signal with only the $F_{1}$ altered. The full-spectrum speech signal was then mixed with pink masking noise and amplified once again prior to presentation over headphones. The total processing delay of the system was $11 \mathrm{~ms}$, regardless of shift magnitude.

For the shifting of $f_{\mathrm{o}}$, the VoiceOne processor was used to alter the line-level (preamplified) microphone signal with no additional filtering. The altered speech signal was then mixed with pink masking noise prior to final amplification and presentation to the participant. The feedback delay in the $f_{\mathrm{o}}$ shifting condition was $9 \mathrm{~ms}$, regardless of shift magnitude. 
The relationship between speech perceptual discrimination and speech production in Parkinson's disease

Under conditions of large $f_{\mathrm{o}}$ perturbation, the DSP increased the $f_{\mathrm{o}}$ by 100 cents $(1$ semitone), for the medium perturbation by 50 cents ( 0.5 semitone), and for the small perturbation by 25 cents $\left(0.25\right.$ semitone). For the large formant perturbation, $F_{1}$ was increased by $30 \%$ (averaging $135.1 \mathrm{~Hz}$ ); for the small formant perturbation, $F_{1}$ was increased $15 \%$ (averaging 47.5 Hz). In PD, different magnitudes of $f_{\mathrm{o}}$ and $F_{1}$ perturbations have been used, and it has been shown that the response to auditory perturbations is not linear (Liu et al., 2012; Chen et al., 2013; Mollaei et al., 2016). Here, in order to be consistent with our previous study, we chose the same magnitudes of $F_{1}$ manipulation and for $f_{\mathrm{o}}$ the medium and the large perturbations were similar to our previous study (Mollaei et al., 2016). However, we added a small magnitude condition (25 cents) to the $f_{\mathrm{o}}$ manipulation based on our pilot testing to hinder any ceiling effect during the auditory discrimination task. The perturbations were measured in cents for $f_{\mathrm{o}}$ because cents are used to express small intervals, and it has been found that healthy adults are capable of recognizing pitch differences as small as 25 cents (Liberman, Cooper, Shankweiler, \& StuddertKennedy, 1967).

In both perturbation conditions $\left(f_{\mathrm{o}}\right.$ and $\left.F_{1}\right)$, the feedback alteration started at the beginning of each trial and lasted for the duration of the trial. The altered speech signal was amplified and presented to participants at a volume of approximately $70 \mathrm{~dB}$ SPL (e.g., Mollaei et al., 2013, 2016). In order to reduce subjects' perception of their unmodified air- and boneconducted speech signal, the subject's altered speech signal was mixed with $60 \mathrm{~dB}$ SPL of pink masking noise. For the listening condition (Experiment 2), we induced the same magnitude of perturbations to pre-recorded samples of speech of the participants, mixed the speech signal with $60 \mathrm{~dB}$ SPL of pink masking noise and presented it back to the participants during the auditory discrimination task. 
The relationship between speech perceptual discrimination and speech production in Parkinson's disease

\subsection{Procedure}

The microphone was placed at a fixed distance of $20 \mathrm{~cm}$ from the mouth. Word production was cued by the presentation of the target word on a computer screen. Participants were instructed to produce the target word "head" that contained the target vowel $[\varepsilon]$ and to prolong the vowel for the duration of 2.5 seconds (guided by the visual presentation of the word on screen) and then to indicate their judgment. Each stimulus was presented for 2.5 seconds followed by a 1.5 second inter-stimulus interval. Participants were asked to begin speaking immediately upon the visual presentation of the target word.

During a practice/setup period (20 trials), participants were asked to produce the target word at a comfortable speaking volume, at which point the microphone gain level was adjusted such that the pre-amplified signal level sent to the vocal processor was close to a known level (i.e., amplification was reduced for relatively loud talkers, and increased for soft talkers). This adjustment yielded a resultant output speech signal of $\sim 70 \mathrm{~dB}$ SPL at the headphones. This was critical to maintain a known speech level relative to the $60 \mathrm{~dB}$ SPL pink masking noise. Along with the individually adjusted microphone gain, a digital VU meter was calibrated for each subject in order to present a visual target to the participant that corresponded to the $70 \mathrm{~dB}$ SPL speech signal level. This VU meter, which was always visible at one edge of the computer monitor directly in front of the subject, presented the desired speech level (+/- $3 \mathrm{~dB})$ as a greencolored target zone. Participants were instructed to maintain their speech level within this zone to the best of their ability, with the experimenter providing verbal feedback (to speak louder or softer) if the level was observed to drift away from the target over several trials. This method has been used in multiple previous studies in both healthy control participants and individuals with 
The relationship between speech perceptual discrimination and speech production in Parkinson's disease

PD (Huang et al., 2019; Mollaei et al., 2013, 2016; Rochet-Capellan \& Ostry, 2011; Shum, Shiller, Baum, \& Gracco, 2011).

After each word production, a question appeared on the computer screen: "Same or different?" to which participants had to respond by indicating on the computer keyboard whether what they heard was the same or different from what they produced. During the listening experiment, participants listened to two speech tokens played one after another and then saw the question on the screen: "Same or different?" to which they responded by pressing the appropriate response key on the computer keyboard. Participants were not instructed to listen to any particular acoustic feature, but rather to simply indicate if the two sounds were different in any way.

\subsection{Data analysis}

For the production trials, data from the first 400 milliseconds (ms) of each vowel production were included in the averaging. For $f_{\mathrm{o}}$-perturbed trials, an auto-correlation method (Zahorian \& Hu, 2008) was used to estimate $f_{\mathrm{o}}$ over a series of overlapping $25 \mathrm{~ms}$ windows of the speech signal (increments of $10 \mathrm{~ms}$ ). The $f_{\mathrm{o}}$ values in $\mathrm{Hz}$ were then converted to the cent scale using the formula: cents $=100 *\left(39.86 * \log 10\left(f_{\mathrm{o}} /\right.\right.$ reference $\left.)\right)$, where reference $=195.997$ $\mathrm{Hz}$ (the note G-3; Chen et al., 2013). For the $F_{1}$-manipulated trials, the $F_{1}$ in the vowel productions in the shifted feedback conditions was identified using a custom-written script in MATLAB (and Signal Processing Toolbox, The MathWorks, Inc., Natick, MA) to carry out linear predictive coding (LPC) acoustic analysis. In extensive prior testing, the software routine was found to provide results highly comparable to that of the LPC-based formant analysis in PRAAT (Burg algorithm) for the same LPC order, pre-emphasis and windowing parameters. The analysis was performed on overlapping $20 \mathrm{~ms}$ windows of the speech signal in increments of 4 
The relationship between speech perceptual discrimination and speech production in Parkinson's disease

ms. Formant estimates for each trial were visually inspected for clearly erroneous values. When the initial formant estimation was a poor fit, the LPC order was manually adjusted to improve the stability of the resulting estimates. The $F_{1}$ trajectory was aligned from the time of the vowel onset and was averaged across trials frame by frame for each condition.

For the measurement of $f_{\mathrm{o}}$ compensation, the magnitude of the vocal response in each perturbation trial was measured as the difference in $f_{\mathrm{o}}$ (at each $10 \mathrm{~ms}$ increment) between the perturbed trial and the immediately preceding, non-perturbed trial. The measurement of $F_{1}$ compensation in each perturbation trial was measured as the difference in $F_{1}$ (at each $4 \mathrm{~ms}$ increment) between the perturbed trial and the immediately preceding, non-perturbed (baseline) trial. The mean $f_{\mathrm{o}}$ and $F_{1}$ compensation traces for each participant, representing the mean change from baseline, were then averaged across participants in each group. Discrimination responses were analyzed in terms of participants' accuracy in detecting the variations in the manipulated signal in the same/different judgment task based on the manipulation type and magnitude by calculating the sensitivity index (d-prime) for each participant and then averaging across participants for each condition. D-prime scores for each participant were calculated by subtracting the z-transform of the hit rate (proportion of stimuli correctly identified as different) from the false alarm rate (proportion of stimuli incorrectly judged as different when they were in fact the same), in order to account for chance guessing (Wickens, 2002).

Statistical analyses were performed using mixed-factorial analyses of variance (ANOVA) with Magnitude (small, medium and large for $f_{0}$, or small and large for $F_{1}$ ) and Task (production and listening) as within-subjects factors and Group (PD and healthy controls) as a betweensubjects factor on the compensation and d-prime scores for $f_{\mathrm{o}}$ and $F_{1}$ separately. Specifically, we were interested in the differences in error detection abilities between individuals with PD and 
The relationship between speech perceptual discrimination and speech production in Parkinson's disease

age-matched control participants, as well as the differences between production and listening tasks in these two groups. Factor and simple effect-sizes were quantified using partial $\eta^{2}$ (Witte $\&$ Witte, 2010) and Pearson's $r$ (Rosnow \& Rosenthal, 1996) based on the amount of variance accounted for by the mixed-factorial ANOVA. Greenhouse-Geisser corrections for unequal variance were applied where warranted. Pearson correlation analyses were carried out between error detection responses (d-prime scores) for $f_{\mathrm{o}}$ or $F_{1}$ across magnitudes for each task and perceptual or clinical rating of severity of PD to investigate the relationship between $f_{\mathrm{o}}$ or $F_{1}$ error detection ability and perceptual or MDS-UPDRS clinical scores in individuals with PD. This resulted in eight correlation analyses. A Bonferroni adjusted alpha rate of 0.006 was used.

In addition, separate Pearson correlation analyses were performed between compensation responses of $f_{\mathrm{o}}$ or $F_{1}$ and error detection responses (d-prime scores in pitch or formant) to investigate the relationship between error correction and detection abilities of individuals with PD. This resulted in ten correlation analyses. A Bonferroni adjusted alpha rate of 0.005 was used.

\section{Results}

\subsection{Experiment One: Production}

For $f_{\mathrm{o}}$, both $\mathrm{PD}$ and control group exhibited a compensatory decrease, opposing the feedback manipulation. The effects of Group (PD vs. control) and Magnitude (small, medium, and large) on $f_{\mathrm{o}}$ compensation were evaluated with a two-way ANOVA. Mean $f_{\mathrm{o}}$ values of the last $100 \mathrm{~ms}$ of each vowel at the three shift magnitudes are shown in Figure 1A. A significant main effect of Magnitude $\left(F[1.52,38.07]=6.96, p<.01\right.$, partial $\left.\eta^{2}=0.22\right)$ and a significant main 
The relationship between speech perceptual discrimination and speech production in Parkinson's disease

effect of Group $\left(F[1,28]=9.09, p<.01\right.$, partial $\left.\eta^{2}=0.24\right)$ were found. The two-way interaction was also significant $\left(F[1.52,38.07]=12.91, p<.05\right.$, partial $\left.\eta^{2}=0.34\right)$. Post hoc simple effects analyses were carried out to examine the difference between groups for each magnitude separately. There was a significant difference between the two groups for the medium $(F[1.52,38.07]=3.03, p<.01, r=0.27)$ and large magnitude perturbations $(F[1.52,38.07]=$ 5.65, $p<.01, r=0.36$ ), with the PD group showing a larger compensatory response. The PD group's response to the small magnitude perturbation was significantly reduced compared to the control group $(F[1.52,38.07]=-4.67, p<.01, r=0.33)$.

For $F_{1}$, the two groups responded to the $F_{1}$ increase with a compensatory decrease in $F_{1}$ output. The effects of Group (PD vs. control) and Magnitude (small vs. large) on $F_{1}$ compensation were evaluated with a two-way ANOVA. Mean $F_{1}$ values of the last $100 \mathrm{~ms}$ of each vowel at the two magnitudes are shown in Figure 1B. A significant main effect of Magnitude (with the large shift showing a greater change than the small shift; $F[1,28]=11.53, p$ $<.01$, partial $\eta^{2}=0.29$ ) and a significant main effect of Group (with the controls showing a greater $F_{1}$ change than the PD group; $F[1,28]=12.23, p<.01$, partial $\eta^{2}=0.30$ ) emerged. The two-way interaction was not significant $(F[1,28]=1.82, p=.19)$.

The means and standard deviations (SD) of d-prime scores during the production condition for pitch and $F_{1}$ shifts of the two groups are shown in Figure 2. The effects of Group (PD and control) and Magnitude (small, medium, and large) for pitch were evaluated using a two-way ANOVA. A significant main effect of Magnitude $\left(F[2,56]=11.99 ; p<.01\right.$, partial $\eta^{2}=$ $0.30)$ and a significant main effect of $\operatorname{Group}\left(F[1,28]=6.51, p<.01\right.$, partial $\left.\eta^{2}=0.19\right)$ were found, with no significant two-way interaction $(F[2,56]=1.12, p=.25)$. Further post hoc simple effects analysis showed a significant difference between small and medium $(F[1,28]=6.15, p<$ 
The relationship between speech perceptual discrimination and speech production in Parkinson's disease

$.01, r=0.42)$ and between small and large magnitude $(F[1,28]=6.03, p<.01, r=0.42)$

conditions with no significant difference between medium and large conditions $(F[1,28]=2.03$, $p=.14)$. The effects of Group (PD and control) and Magnitude (small and large) for $F_{1}$ modulation were also evaluated using a two-way ANOVA. A significant main effect of Magnitude $\left(F[1,28]=30.96, p<.01\right.$, partial $\left.\eta^{2}=0.52\right)$ was noted with no significant main effect of $\operatorname{Group}(F[1,28]=0.16, p=.71)$ or two-way interaction $(F[1,28]=.003, p=.94)$.

A further analysis focused on the relationship between the degree of compensation (last $100 \mathrm{~ms}$ of compensation) and self-monitoring accuracy in the production of $f_{\mathrm{o}}$ and $F_{1}$ conditions at each magnitude in both groups. No significant correlations were found for any of the magnitudes for $f_{\mathrm{o}}$ (small magnitude: $r=-0.01, p=.96$; medium magnitude: $r=-0.21, p=.46$; large magnitude: $r=-0.23, p=.41$ ) or $F_{1}$ (small magnitude: $r=0.11, p=.71$; large magnitude: $r$ $=0.19, p=.50)$.

\subsection{Experiment Two: Listening}

During listening for differences in $f_{\mathrm{o}}$, a significant main effect of Magnitude $(F[2,56]=$ 98.55; $p<.01$, partial $\left.\eta^{2}=0.78\right)$ was found with no significant main effect of $\operatorname{Group}(F[1,28]=$ $0.41, p=.52)$ or two-way interaction $(F[2,56]=0.52, p=.59)$. A post hoc analysis focused on the differences among the three magnitudes across both PD and control groups. Significant differences between the small and medium $(F[1,28]=52.08 ; p<.01)$, small and large $(F[1,28]=$ 43.66; $p<.01)$, and medium and large magnitude manipulations $(F[1,28]=59.49 ; p<.01)$ were found across groups (Figure 3A).

For the detection of $F_{1}$ differences, a significant main effect of Magnitude $(F[1,28]=$ 18.46; $p<.01$, partial $\eta^{2}=0.40$ ) and a significant main effect of Group (with controls showing greater sensitivity than the PD group; $F[1,28]=7.90 ; p<.01$, partial $\eta^{2}=0.22$ ) were found, with 
The relationship between speech perceptual discrimination and speech production in Parkinson's disease

no significant two-way interaction $(F[1,28]=0.002, p=.94)$, as shown in Figure 3B.

A further analysis focused on the relationship between the degrees of compensation to $f_{\mathrm{o}}$ or $F_{1}$ manipulation and the error detection accuracy in the listening task. No significant correlations were found for any of the magnitudes for $f_{\mathrm{o}}$ (small magnitude: $r=-0.16, p=.58$; medium magnitude: $r=0.23, p=.40$; large magnitude: $r=0.15, p=.58$ ) and $F_{1}$ (small magnitude: $r=0.04, p=.88$; large magnitude: $r=0.08, p=.76$ ).

\subsection{Differences in detection between production and listening}

The effects of Task (production vs. listening) and Group (control participants and participants with PD) were evaluated with a two-way ANOVA for each manipulation condition $\left(f_{\mathrm{o}}\right.$ and $\left.F_{1}\right)$ separately across perturbations magnitudes (Figure 4$)$. For the detection of $f_{\mathrm{o}}$ changes, there was no main effect of Group $(F[1,28]=0.92 ; p=.34)$ and no significant main effect of $\operatorname{Task}(F[1,28]=0.03 ; p=.85)$ and no two-way interaction $(F[1,28]=0.45 ; p=.50)$. For the detection of $F_{1}$ changes, a significant main effect of Task $\left(F[1,28]=70.74 ; p<.01\right.$, partial $\eta^{2}=$ $0.72)$, and a significant two-way interaction $\left(F[1,28]=4.70 ; p<.05\right.$, partial $\left.\eta^{2}=0.14\right)$ was found but there was no significant main effect of $\operatorname{Group}(F[1,28]=3.49 ; p=.07)$. Participants across groups detected speech errors during the listening task significantly more accurately than during the production task (mean \pm standard deviation: $2.73 \pm 0.76$ vs. $1.27 \pm 0.61$ ).

\subsection{Relationship with clinical ratings of severity}

We investigated the relationship between the degree of severity of either the total dysarthria perceptual score or MDS-UPDRS clinical rating score and the ability to detect changes in $f_{\mathrm{o}}$ or $F_{1}$ in the $\mathrm{PD}$ group (Table 2) using Pearson correlation analysis. For this analysis, we combined the error detection results across all the magnitudes for each manipulation 
The relationship between speech perceptual discrimination and speech production in Parkinson's disease

condition. None of the correlations reached significance using Bonferroni correction for multiple comparisons.

\section{Discussion}

Two experiments were conducted to assess the effects of auditory feedback alterations on speech production and speech perception of participants with PD. For the first study, the compensation paradigm was similar to our previous study and participants with PD responded with a greater compensatory response to medium and large $f_{\mathrm{o}}$ feedback perturbations while $F_{1}$ feedback perturbation resulted in a significant reduction in compensation compared to controls (Mollaei et al., 2016). The current results thus confirm a differential effect of PD on auditory feedback parameters during speech production. For the second study, we examined the ability of individuals with PD to detect their own speech changes immediately following production and under listening conditions, in which judgments were made from samples recorded during the first experiment. Interestingly, different patterns emerged across the two experiments and, importantly, for the two acoustic parameters $\left(f_{\mathrm{o}}\right.$ and $\left.F_{1}\right)$. During self-monitoring while speaking, participants with PD appeared to be more sensitive than control participants to manipulations of $f_{\mathrm{o}}$, but not $F_{1}$. However, the results of the listening task revealed that participants with PD were less sensitive than control participants to manipulations of $F_{1}$. Possible explanations of these patterns in the context of what is known about speech processing in PD will be outlined in what follows.

\subsection{Comparison of findings across tasks: pitch}

During speech production, participants with PD showed an increased compensatory 
The relationship between speech perceptual discrimination and speech production in Parkinson's disease

response to $f_{\mathrm{o}}$ changes (with the exception of the small magnitude $f_{\mathrm{o}}$ perturbations). This is consistent with prior findings of enhanced responses to $f_{\mathrm{o}}$ perturbations reported in the literature (Chen et al., 2013; Huang et al., 2016; Liu et al., 2012; Mollaei et al., 2016). Participants with PD also demonstrated increased perceptual sensitivity to pitch shifts during production, and a trend towards increased sensitivity during listening. This increased sensitivity during production may be related to the observation that while individuals with PD speak with reduced pitch and loudness modulation, they report their speech to be produced with normal variation in pitch and loudness. However, the response to the small pitch perturbation ( 25 cents) was reduced in PD compared to the control group. It has been reported previously that response to pitch perturbation is related to the shift magnitude, and the auditory system is optimally tuned to compensate for small magnitudes of shift compared to large shifts in healthy control participants (Liu \& Larson, 2007). The auditory system is capable of detecting changes as small as 10 cents in magnitude and the auditory-motor system is capable of compensating for 10 cents pitch shift (Liu \& Larson, 2007). The normal variation in pitch compensation is somewhat opposite of what we observed here in PD, in that for pitch shifts of 25 cents and below, the percent response magnitude is around 100\% (Larson, Burnett, Bauer, Kiran, \& Hain, 2001), and for above 25 cents shifts, the percent response magnitude is below 50\% (Kiran \& Larson, 2001). In two previous studies using a pitch compensation paradigm in PD, two different magnitudes of shift (50 cents and 100 cents) were used (Chen et al., 2013; Mollaei et al., 2016), and they also showed greater sensitivity (gain in response) to the smaller 50 cents shift. The reduced response to the 25 cents shift found in the present study suggests either that subtle changes in pitch may not be perceived in PD due to reduced sensitivity to normal variation or their response to the small shifts in pitch are more variable, reducing the overall mean response, as observed in Figure 1A, with high standard 
The relationship between speech perceptual discrimination and speech production in Parkinson's disease

deviations for the small shift in PD compared to the control group.

The perception of loudness during speech production has been studied more than any other speech parameter in PD and has been shown to be affected by PD (Brajot et al., 2016; Clark et al., 2014; De Keyser et al., 2016; Fox \& Ramig, 1997; Ho et al., 2000; Kwan \& Whitehill, 2011). Clark et al. (2014) asked participants with PD to produce speech at normal loudness, two, and four times louder and quieter than the normal loudness level. They observed that participants with PD overestimated their own loudness, and when they were asked to make proportional changes in their loudness, they made relatively smaller adjustments in speech intensity despite having adequate capacity to do so. De Keyser et al. (2016) suggested that auditory perceptual deficits may influence speech production in individuals with PD based on their findings from intensity estimation and imitation tasks. They found that individuals with PD had a flatter slope in intensity imitation and a restricted range in intensity estimation compared with control participants. These results suggest abnormal self-monitoring of loudness during speech that may be caused by increased sensitivity at the cortical and/or subcortical level to the transient acoustic parameters of speech such as amplitude and pitch.

During vocalization, vocal fold vibration gives rise to multiple, harmonically related spectral components that create redundancy and enhance the perception of pitch, but not formants. In addition, laryngeal somatosensory information during vocalization(Gozaine \& Clark, 2005; Larson, Altman, Liu, \& Hain, 2008) adds to the perception of pitch changes compared to the perception of formant changes. Furthermore, the enhanced speech production response to pitch alterations but not formants reported here and previously observed (Chen et al., 2013; Huang et al., 2016; Liu et al., 2012; Mollaei et al., 2016) may be partially accounted for by an increased auditory sensitivity to frequencies that fall within the range of $f_{\mathrm{o}}$. The basal ganglia 
The relationship between speech perceptual discrimination and speech production in Parkinson's disease

have been associated with gating sensory information by filtering relevant from irrelevant information, and it seems that in PD this gating is affected (Haslinger et al., 2001; Liotti et al., 2003; Schneider, Diamond, \& Markham, 1987) during speech production as well as speech perception. Moreover, as initial pitch processing occurs at the level of the brainstem (Aitkin, 1986; Plack, Barker, \& Hall, 2014), the enhanced compensatory response to pitch perturbation and increased sensitivity to pitch detection may reflect a release from inhibition due to reduced brainstem dopamine in PD (Nevue, Elde, Perkel, \& Portfors, 2016a; Nevue, Felix, \& Portfors, 2016b).

Our results suggest that individuals with PD are more sensitive to pitch-related auditory input during both production and perception, for shifts of 50 cents or more. For shifts of 25 cents and below, there may be a reduced sensitivity in detecting and/or correcting for subtle pitch changes during production. The possible deficit associated with somatosensory information in Parkinson's dysarthria (Hammer \& Barlow, 2010) and auditory gating deficits may contribute to an increase in sensitivity of the auditory system to $f_{\mathrm{o}}$ by altering the balance between the auditory and somatosensory systems at the level of the brainstem where the neural firing rate encodes pitch (Plack et al., 2014). Further investigation is required to clarify the possible role of brainstem dopamine circuits in pitch and loudness processing in PD.

\subsection{Comparison of findings across tasks: formants}

The detection accuracy for changes in $F_{1}$ was more robust during listening compared to self-monitoring during production for both groups. As has been observed previously from electroencephalographic and magnetoencephalographic recordings, the evoked neural response from self-generated auditory feedback is reduced when compared to listening of the recorded vocalizations from the same participants - the so-called speech induced suppression (SIS) effect 
The relationship between speech perceptual discrimination and speech production in Parkinson's disease

(Heinks-Maldonado Mathalon, Gray, \& Ford, 2005; Houde, Nagarajan, Sekihara, \& Merzenich, 2002; Tourville et al., 2008). This suppression effect observed cortically likely reflects a reduced sensitivity to aspects of the auditory input during the production task compared to listening, thereby reducing the capacity overall to identify subtle formant differences. In addition, the acoustic features of the manipulation (involving only $F_{1}$, and none of the higher formants), would lead to a less perceptually salient cue compared to $f_{\mathrm{o}}$ shift. These factors may have combined to make the self-monitoring of formant changes more difficult than for $f_{\mathrm{o}}$ changes for listeners in both PD and control groups. Interestingly, in this study at the behavioural level, there was no SIS effect observed for $f_{\mathrm{o}}$ detection under the active listening relative to the passive listening condition consistent with a differential modulation of laryngeal pitch compared to the articulatory acoustic parameters.

In contrast to speech production, during listening, the control group demonstrated a better capacity to detect the same subtle formant differences than participants with PD. The difference in detection performance for formant changes during the listening task may reflect a sensory deficit related to more subtle acoustic manipulations in PD. One study that used the same discrimination paradigm as the current study to detect amplitude and frequency of pure tones found reduced detection ability in PD compared to healthy controls (Troche et al., 2012). However, there are a number of studies that tested loudness and amplitude perception and did not find any differences in the loudness growth slope (Abur et al., 2018b) or loudness rating (Dromey \& Adams, 2000). In addition, Huang et al. (2016) found no differences at the cortical level between individuals with PD and control participants when participants were listening to the frequency altered feedback of their pre-recorded speech. It should be pointed out that these studies used different methodologies to test loudness perception and their inclusion criteria also 
The relationship between speech perceptual discrimination and speech production in Parkinson's disease

differed slightly (e.g., in hearing status). For example, Abur et al. (2018b) based their

suggestions on a loudness perceptual judgment task for pure tones presented through insert

earphones. They included hearing thresholds of $25 \mathrm{~dB}$ HL for $1000 \mathrm{~Hz}$ and below and $35 \mathrm{~dB} \mathrm{HL}$

for frequencies above $1000 \mathrm{~Hz}$ (Abur et al., 2018b). Dromey and Adams (2000) did not use a

hearing screening but tested participants' loudness perception for tones using a magnitude

estimation task presented through a loudspeaker at different sound pressure levels. They then

correlated participants' hearing thresholds with loudness ratings and found no significant

correlation. Huang et al. (2016) used a passive listening task that included pitch-shifted

recordings of participants' speech presented through insert earphones, and they included hearing

thresholds of $40 \mathrm{~dB}$ HL or less for 500, 1000, 2000, and $4000 \mathrm{~Hz}$. Abur et al. (2018b) and

Dromey and Adams (2000) tested individuals with PD on medication; however, in our study, individuals with PD were off medication for 12 hours. The effect of medication on speech perception and production in PD certainly requires further investigation. The current findings indicate that individuals with PD have speech monitoring deficits at least for the articulatory parameter of $F_{1}$. The findings also point to the heterogeneous effects of the disorder on error monitoring for pitch vs. $F_{1}$.

\subsection{Relationship of compensation responses and severity to detection accuracy}

No correlation was found between detection accuracy during speech self-monitoring or listening and compensation responses in both $f_{\mathrm{o}}$ and $F_{1}$ consistent with recent findings of unimpaired verbal monitoring in PD, at least at the single word level (Gauvin et al., 2017; however, see also McNamara et al., 1992). However, the lack of correlation between speech production and perception system may be due to the small sample size in the current study. Additionally, we did not find any relationship between detection accuracy across all perturbation 
The relationship between speech perceptual discrimination and speech production in Parkinson's disease

magnitudes in $f_{\mathrm{o}}$ or $F_{1}$ and disease severity in MDS-UPDRS or perceptual dysarthria scores using total speech subsystems. These non-significant results are consistent with the findings from our prior study in the relationship between $f_{\mathrm{o}}$ or $F_{1}$ compensation responses and MDS-UPDRS or perceptual dysarthria scores (Mollaei et al., 2016).

\subsection{Limitations and future directions}

The error detection of $f_{\mathrm{o}}$ in the listening task showed a trend towards better performance in PD but the trend was not statistically significant, most likely due to the small sample size. In addition, the heterogeneous nature of the disorder and the state of the participants with PD at the time of testing limit the ability to fully interpret differences due to disease severity. Individuals with PD showed a wide range in MDS-UPDRS motor scores (3-48). It would be useful in future studies to increase the sample size to obtain a better distribution of severity to assess the effects of disease severity on perceptual processing. Although participants were tested off medication for 12 hours, based on disease severity and the L-dopa dosage, it is difficult to determine the degree of 'on' or 'off' state of PD symptoms, as it does not have a linear relationship with medication intake (Fern-Pollak, Whone, Brooks, \& Mehta, 2004). As such, evaluating symptom severity immediately prior to the onset of testing may provide a better indication of the state of the disease at the time of testing. In addition, the MDS-UPDRS was assessed by the first author who received in-house training but did not hold official certification for the examination. This may have affected its reliability, although a second trained rater provided comparable scores.

\subsection{Conclusion}

The current results and previous findings support the hypothesis that individuals with PD demonstrate differential perceptual sensitivity to speech acoustic parameters. Overall, the results 
The relationship between speech perceptual discrimination and speech production in Parkinson's disease

of the present investigation provide insight into the production and perceptual deficits in PD. The increased detection accuracy for pitch while producing speech and reduced detection accuracy for $F_{1}$ during listening further highlight the differential effects of the disease on speech perception and point to speech error monitoring deficits in PD. The lack of correlation between compensation responses and error monitoring ability in PD makes it difficult to relate the speech production deficits to perceptual disorders; however, including a larger sample size in future studies may help to pinpoint the relationship of speech perception and speech production deficits in PD. In addition, future investigation should focus on the exact neurobiological underpinnings of these auditory perceptual deficits, specifically increased sensitivity to pitch changes in PD. 
The relationship between speech perceptual discrimination and speech production in Parkinson's disease

Acknowledgement: The authors would like to thank all the participants who took part in this study. We also would like to thank Suzanne Lalonde, SLP(C) and Francois-Xavier Brajot, PhD, CCC-SLP, for their help with data analysis. 
The relationship between speech perceptual discrimination and speech production in Parkinson's disease

\section{References}

Abur, D., Lester-Smith, R. A., Daliri, A., Lupiani, A. A., Guenther, F. H., \& Stepp, C. E. (2018a). Sensorimotor adaptation of voice fundamental frequency in Parkinson's disease. PLOS One. 13(1), e0191839.

Abur, D., Lupiani, A. A., Hickox, A. E., Shinn-Cunningham, B. G., \& Stepp, C. E. (2018b). Loudness Perception of Pure Tones in Parkinson's Disease. Journal of Speech, Language, and Hearing Research, 61(6), 1487-1496.

Aitkin, L. (1986). The auditory midbrain: structure and function in the central auditory pathway. New York, New York: Springer Science \& Business Media. pp 24-30.

Arnold, C., Gehrig, J., Gispert, S., Seifried, C., \& Kell, C. A. (2014). Pathomechanisms and compensatory efforts related to Parkinsonian speech. NeuroImage: Clinical, 4, 82-97.

Behroozmand, R., Shebek, R., Hansen, D. R., Oya, H., Robin, D. A., Howard III, M. A., \& Greenlee, J. D. (2015). Sensory-motor networks involved in speech production and motor control: An fMRI study. Neuroimage, 109, 418-428.

Brajot, F. X., Shiller, D. M., \& Gracco, V. L. (2016). Autophonic loudness perception in Parkinson's disease. The Journal of the Acoustical Society of America, 139(3), 1364-1371.

Brown, R. G., \& Marsden, C. D. (1988). Internal versus external cues and the control of attention in Parkinson's disease. Brain, 111, 323-345. doi:10.1093/brain/111.2.323

Chen, X., Zhu, X., Wang, E. Q., Chen, L., Li, W., Chen, Z., \& Liu, H. (2013). Sensorimotor control of vocal pitch production in Parkinson's disease. Brain Research,1527, 99-107.

Cicchetti, D. V. (1994). Guidelines, criteria, and rules of thumb for evaluating normed and standardized assessment instruments in psychology. Psychological assessment, 6(4), 284.

Clark, J. P., Adams, S. G., Dykstra, A. D., Moodie, S., \& Jog, M. (2014). Loudness perception and speech intensity control in Parkinson's disease. Journal of communication disorders, 51, 112.

Cowie, R., \& Douglas-Cowie, E. (1992). Postlingually Acquired Deafness: Speech Deterioration and the Wider Consequences. New York, New York: Mouton de Gruyter.

Darley, F. L., Aronson, A. E., \& Brown, J. R. (1969). Clusters of deviant speech dimensions in the dysarthrias. Journal of speech and hearing research, 12(3), 462-496.

De Keyser, K., Santens, P., Bockstael, A., Botteldooren, D., Talsma, D., De Vos, S., ... \& De Letter, M. (2016). The relationship between speech production and speech perception deficits in parkinson's disease. Journal of Speech, Language, and Hearing Research, 59(5), 915-931. 
The relationship between speech perceptual discrimination and speech production in Parkinson's disease

Dromey, C., \& Adams, S. (2000). Loudness perception and hypophonia in Parkinson disease. Journal of Medical Speech-Language Pathology, 8(4), 255-259.

Duffy, J. R. (2013). Motor speech disorders: Substrates, differential diagnosis, and management. St. Louis, MO: Elsevier Mosby. pp 198-217.

Fahn, S., Elton, R. L., \& Committee, U. D. (1987). Unified Parkinson's disease rating scale. In Fahn, S., Marsden, C. D., Calne, D. B., Goldstein, M. (Eds), Recent Development in Parkinson's Disease. Floral Park, NJ: Macmillan Healthcare Information. pp 293-304.

Fairbanks, G. (1960). Voice and articulation drillbook (Vol. 127). New York: Harper.

Fern-Pollak, L., Whone, A. L., Brooks, D. J., \& Mehta, M. A. (2004). Cognitive and motor effects of dopaminergic medication withdrawal in Parkinson's disease. Neuropsychologia, 42(14), 1917-1926.

Fox, C. M., \& Ramig, L. O. (1997). Vocal sound pressure level and self-perception of speech and voice in men and women with idiopathic Parkinson disease. American Journal of SpeechLanguage Pathology, 6(2), 85-94. doi:10.1044/1058-0360.0602.85.

Gauvin, H. S., Mertens, J., Mariën, P., Santens, P., Pickut, B. A., \& Hartsuiker, R. J. (2017). Verbal monitoring in Parkinson's disease: A comparison between internal and external monitoring. PloS one, 12(8), e0182159.

Gozaine, T. C., \& Clark, K. F. (2005). Function of the laryngeal mechanoreceptors during vocalization. The Laryngoscope, 115(1), 81-88.

Hammer, M. J., \& Barlow, S. M. (2010). Laryngeal somatosensory deficits in Parkinson's disease: implications for speech respiratory and phonatory control. Experimental Brain Research, 201(3), 401-409.

Haslinger, B., Erhard, P., Kämpfe, N., Boecker, H., Rummeny, E., Schwaiger, M., ... \& Ceballos-Baumann, A. O. (2001). Event-related functional magnetic resonance imaging in Parkinson's disease before and after levodopa. Brain, 124(3), 558-570.

Heinks-Maldonado, T. H., Mathalon, D. H., Gray, M., \& Ford, J. M. (2005). Fine-tuning of auditory cortex during speech production. Psychophysiology, 42(2), 180-190.

Ho, A. K., Bradshaw, J. L., \& Iansek, R. (2000). Volume perception in parkinsonian speech. Movement Disorders, 15, 1125-1131. doi:10.1002/1531-8257(200011)15:6<1125::aid-mds1010.

Ho, A. K., Iansek, R., Marigliani, C., Bradshaw, J. L., \& Gates, S. (1999). Speech impairment in a large sample of patients with Parkinson's disease. Behavioural neurology, 11(3), 131-137. 
The relationship between speech perceptual discrimination and speech production in Parkinson's disease

Houde, J. F., Nagarajan, S. S., Sekihara, K., \& Merzenich, M. M. (2002). Modulation of the auditory cortex during speech: an MEG study. Journal of cognitive neuroscience, 14(8), 11251138.

Huang, X., Chen, X., Yan, N., Jones, J. A., Wang, E. Q., Chen, L., ... \& Liu, H. (2016). The impact of parkinson's disease on the cortical mechanisms that support auditory-motor integration for voice control. Human brain mapping, 37(12), 4248-4261.

Huang, X., Fan, H., Li, J., Jones, J. A., Wang, E. Q., Chen, L., ... \& Liu, H. (2019). External cueing facilitates auditory-motor integration for speech control in individuals with Parkinson's disease. Neurobiology of aging, 76, 96-105.

Kiran, S., \& Larson, C. R. (2001). Effect of duration of pitch-shifted feedback on vocal responses in patients with Parkinson's disease. Journal of Speech, Language, and Hearing Research.

Kwan, L. C., \& Whitehill, T. L. (2011). Perception of speech by individuals with Parkinson's disease: A review. Parkinson's Disease, 2011.

Larson, C. R., Altman, K. W., Liu, H., \& Hain, T. C. (2008). Interactions between auditory and somatosensory feedback for voice $F_{0}$ control. Experimental Brain Research, 187(4), 613-621.

Larson, C. R., Burnett, T. A., Bauer, J. J., Kiran, S., \& Hain, T. C. (2001). Comparison of voice F 0 responses to pitch-shift onset and offset conditions. The Journal of the Acoustical Society of America, 110(6), 2845-2848.

Larson, C. R., Burnett, T. A., Kiran, S., \& Hain, T. C. (2000). Effects of pitch-shift velocity on voice F 0 responses. The Journal of the Acoustical Society of America, 107(1), 559-564.

Liberman, A. M., Cooper, F. S., Shankweiler, D. P., \& Studdert-Kennedy, M. (1967). Perception of the speech code. Psychological review, 74(6), 431.

Liotti, M., Ramig, L. O., Vogel, D., New, P., Cook, C. I., Ingham, R. J., ... \& Fox, P. T. (2003). Hypophonia in Parkinson's disease neural correlates of voice treatment revealed by PET. Neurology, 60(3), 432-440.

Liu, H., Auger, J., \& Larson, C. R. (2010). Voice fundamental frequency modulates vocal response to pitch perturbations during English speech. The Journal of the Acoustical Society of America, 127(1), EL1-EL5.

Liu, H., \& Larson, C. R. (2007). Effects of perturbation magnitude and voice F 0 level on the pitch-shift reflex. The Journal of the Acoustical Society of America, 122(6), 3671-3677.

Liu, H., Wang, E. Q., Metman, L. V., \& Larson, C. R. (2012). Vocal responses to perturbations in voice auditory feedback in individuals with Parkinson's disease. PLOS One, 7, e33629. 
The relationship between speech perceptual discrimination and speech production in Parkinson's disease

MacDonald, E. N., Purcell, D. W., \& Munhall, K. G. (2011). Probing the independence of formant control using altered auditory feedback. The Journal of the Acoustical Society of America, 129(2), 955-965.

Macmillan, N. A., \& Creelman, C. D. (2005). Detection Theory: A User's Guide. Mahwah, New Jersey: Lawrence Earlbaum Associates.

McNamara, P., Obler, L. K., Au, R., Durso, R., \& Albert, M. L. (1992). Speech monitoring skills in Alzheimer's disease, Parkinson's disease, and normal aging. Brain and Language, 42(1), 3851.

Mollaei, F., Shiller, D. M., Baum, S. R., \& Gracco, V. L. (2016). Sensorimotor control of vocal pitch and formant trajectories in Parkinson's disease. Brain Research, 1646, 269-277.

Mollaei, F., Shiller, D. M., \& Gracco, V. L. (2013). Sensorimotor adaptation of speech in Parkinson's disease. Movement Disorders, 28(12), 1668-1674. doi.org/10.1002/mds.2558.

Nasreddine, Z. S., Phillips, N. A., Bédirian, V., Charbonneau, S., Whitehead, V., Collin, I., ... \& Chertkow, H. (2005). The Montreal Cognitive Assessment, MoCA: a brief screening tool for mild cognitive impairment. Journal of the American Geriatrics Society, 53(4), 695-699.

Nevue, A. A., Elde, C. J., Perkel, D. J., \& Portfors, C. V. (2016a). Dopaminergic input to the inferior colliculus in mice. Frontiers in neuroanatomy, 9, 168.

Nevue, A. A., Felix II, R. A., \& Portfors, C. V. (2016b). Dopaminergic projections of the subparafascicular thalamic nucleus to the auditory brainstem. Hearing research, 341, 202-209.

Niziolek, C. A., Nagarajan, S. S., \& Houde, J. F. (2013). What does motor efference copy represent? Evidence from speech production. Journal of Neuroscience, 33(41), 16110-16116.

Perkell, J. S., Guenther, F. H., Lane, H., Matthies, M. L., Perrier, P., Vick, J., ... \& Zandipour, M. (2000). A theory of speech motor control and supporting data from speakers with normal hearing and with profound hearing loss. Journal of Phonetics, 28(3), 233-272.

Perkell, J., Matthies, M., Lane, H., Guenther, F., Wilhelms-Tricarico, R., Wozniak, J., \& Guiod, P. (1997). Speech motor control: Acoustic goals, saturation effects, auditory feedback and internal models. Speech communication, 22(2-3), 227-250.

Plack, C. J., Barker, D., \& Hall, D. A. (2014). Pitch coding and pitch processing in the human brain. Hearing Research, 307, 53-64.

Purcell, D. W., \& Munhall, K. G. (2006). Compensation following real-time manipulation of formants in isolated vowels. The Journal of the Acoustical Society of America, 119(4), 22882297. 
The relationship between speech perceptual discrimination and speech production in Parkinson's disease

Rochet-Capellan, A., \& Ostry, D. J. (2011). Simultaneous acquisition of multiple auditory-motor transformations in speech. Journal of Neuroscience, 31(7), 2657-2662.

Rosnow, R. L., \& Rosenthal, R. (1996). Computing contrasts, effect sizes, and counternulls on other people's published data: General procedures for research consumers. Psychological methods, 1(4), 331.

Roy, N., Nissen, S. L., Dromey, C., \& Sapir, S. (2009). Articulatory changes in muscle tension dysphonia: evidence of vowel space expansion following manual circumlaryngeal therapy. Communications Disorders, 42(2), 124-135.

Schneider, J. S., Diamond, S. G., \& Markham, C. H. (1987). Parkinson's disease Sensory and motor problems in arms and hands. Neurology, 37(6), 951-951.

Shum, M., Shiller, D. M., Baum, S. R., \& Gracco, V. L. (2011). Sensorimotor integration for speech motor learning involves the inferior parietal cortex. European Journal of Neuroscience, 34(11), 1817-1822.

Skodda, S., Groenheit, W., \& Schlegel, U. (2012). Impairment of vowel articulation as a possible marker of disease progression in Parkinson's disease. PLOS One, 7(2). doi:10.1371/journal. pone. 0032132

Skodda, S., Rinsche, H., \& Schlegel, U. (2009). Progression of dysprosody in Parkinson's disease over time: A longitudinal study. Movement Disorders, 24, 716-722. doi:10.1002/mds.22430

Skodda, S., Visser, W., \& Schlegel, U. (2011a). Vowel articulation in Parkinson's disease. Journal of Voice, 25, 467-472. doi:10.1016/ j.jvoice.2010.01.009.

Skodda, S., Visser, W., \& Schlegel, U. (2011b). Gender-related patterns of dysprosody in Parkinson disease and correlation between speech variables and motor symptoms. Journal of Voice, 25(1), 76-82.

Svirsky, M. A., Lane, H., Perkell, J. S., \& Wozniak, J. (1992). Effects of short-term auditory deprivation on speech production in adult cochlear implant users. The Journal of the Acoustical Society of America, 92(3), 1284-1300.

Troche, J., Troche, M. S., Berkowitz, R., Grossman, M., \& Reilly, J. (2012). Tone discrimination as a window into acoustic perceptual deficits in Parkinson's disease. American Journal of Speech-Language Pathology, 21(3), 258-263.

Tourville, J. A., Reilly, K. J., \& Guenther, F. H. (2008). Neural mechanisms underlying auditory feedback control of speech. Neuroimage, 39(3), 1429-1443.

Wickens, T. D. (2002). Elementary signal detection theory. New York, Oxford University Press, USA. pp 3-16. 
The relationship between speech perceptual discrimination and speech production in Parkinson's disease

Witte, R., \& Witte, J. (2010). Statistics. Hoboken, NJ: J.

Zahorian, S. A., \& Hu, H. (2008). A spectral/temporal method for robust fundamental frequency tracking. The Journal of the Acoustical Society of America, 123(6), 4559-4571. 
The relationship between speech perceptual discrimination and speech production in Parkinson's disease

\section{Figure Legends}

Figure 1. A: Compensation to $f_{\mathrm{o}}$ perturbation with three magnitudes (small: 25 cents, medium: 50 cents, large: 100 cents) in individuals with PD (yellow) and control participants (blue), B: Compensation to $F_{1}$ perturbation with two magnitudes (small: $47.5 \mathrm{~Hz}$, large: $135.1 \mathrm{~Hz}$ ) in individuals with PD (yellow), control participants (blue). Error bars show the standard deviation (SD). Note: $* p<.01$

Figure 2. A: The mean of averaged d-prime scores of $f_{\mathrm{o}}$ errors (small: 25 cents, medium: 50 cents, large: 100 cents) in PD (yellow) and controls (blue) during production (left), B: Mean of d-prime scores of $F_{1}$ errors (small: $47.5 \mathrm{~Hz}$, large: $135.1 \mathrm{~Hz}$ ) in PD (yellow) and controls (blue) during production (right). Error bars show standard deviation (SD). Note: ${ }^{*} p<.01$

Figure 3. A: The mean of averaged of d-prime scores of $f_{\mathrm{o}}$ errors (small: 25 cents, medium: 50 cents, large: 100 cents) in PD (yellow) and controls (blue) during listening (left), B: Mean of dprime scores of $F_{1}$ errors (small: $47.5 \mathrm{~Hz}$, large: $135.1 \mathrm{~Hz}$ ) in PD (yellow) and controls (blue) during listening (right). Error bars show standard deviation (SD). Note: ${ }^{*} p<.01$

Figure 4. The mean of averaged d-prime scores in response to $f_{\mathrm{o}}$ errors (left) and in response to $F_{1}$ errors (right) in control participants (blue) and participants with PD (yellow) during production and listening. Error bars show the standard deviation (SD). 
The relationship between speech perceptual discrimination and speech production in Parkinson's disease

Figure 1.

A

Compensation to $f_{\mathrm{o}}$ perturbation

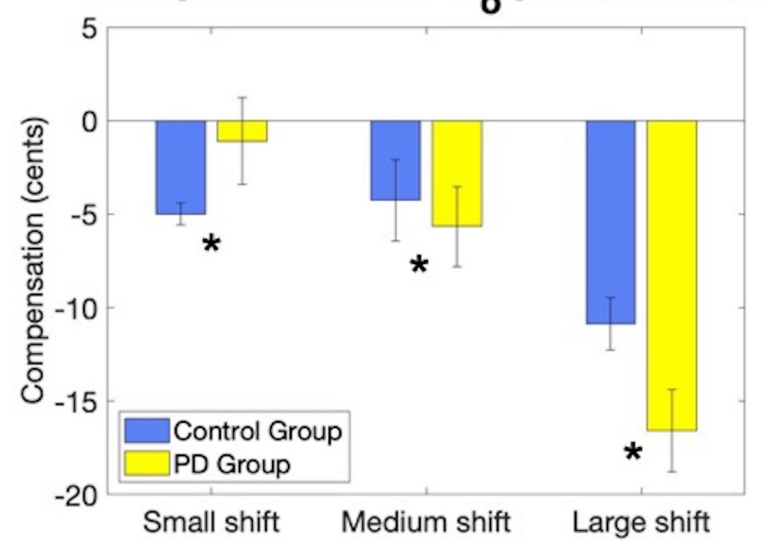

Note: ${ }^{*} p<0.01$
B

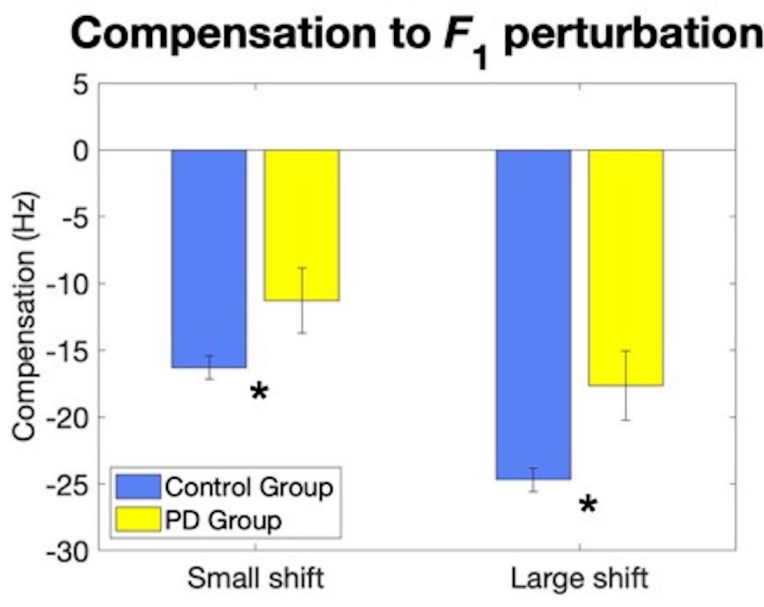

Figure 2.

A

Production: $\boldsymbol{f}_{\mathrm{o}}$

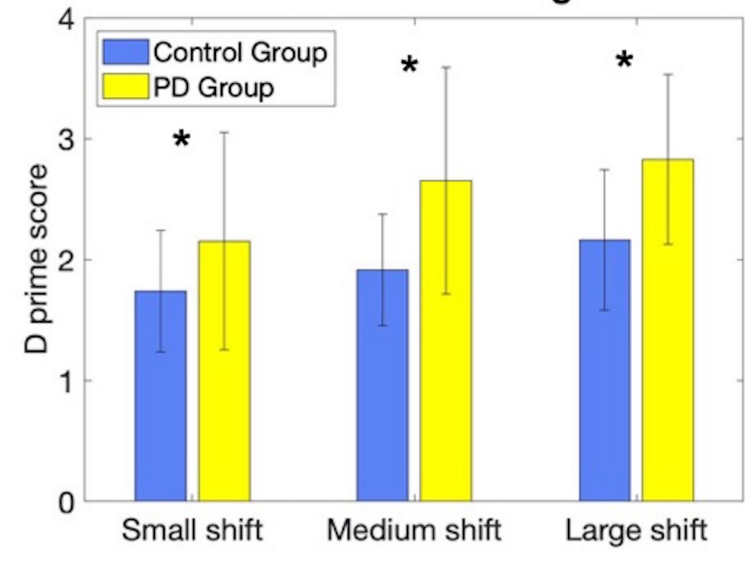

Note: ${ }^{*} p<0.01$

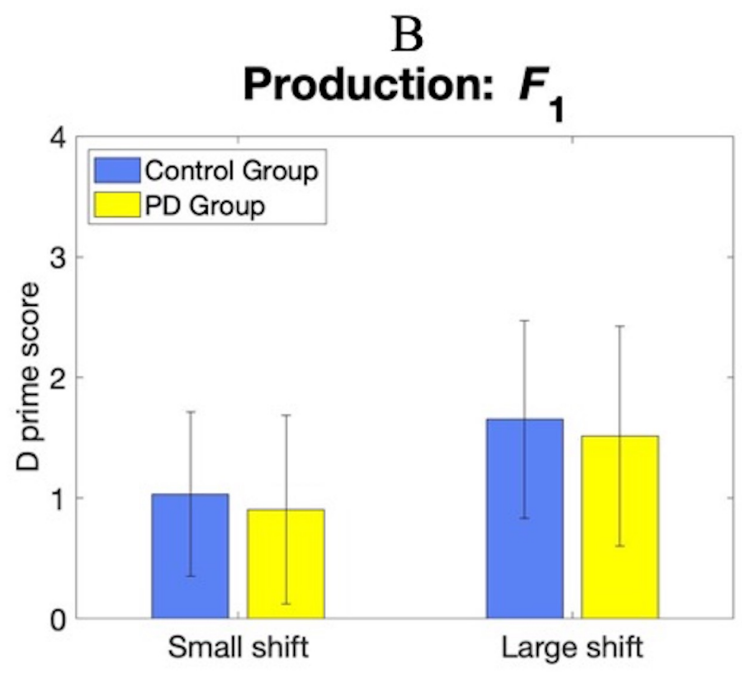


The relationship between speech perceptual discrimination and speech production in Parkinson's disease

\section{Figure 3.}

A

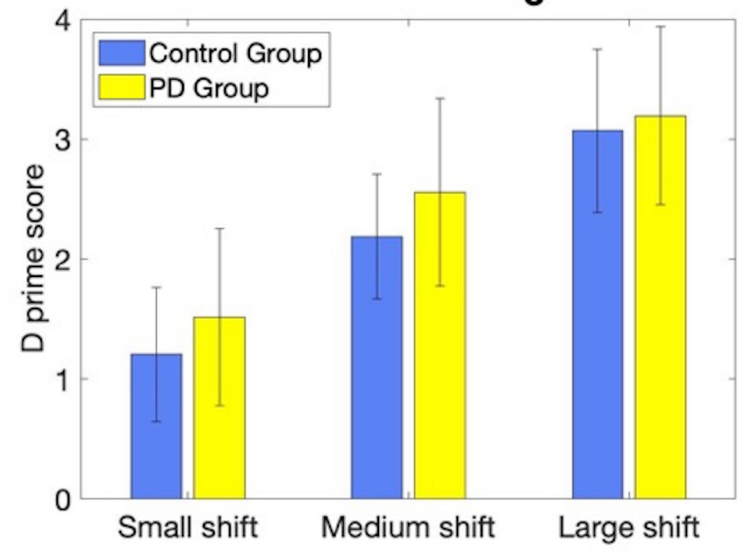

Note: ${ }^{*} p<0.01$
Listening: $\boldsymbol{f}_{\mathrm{o}}$

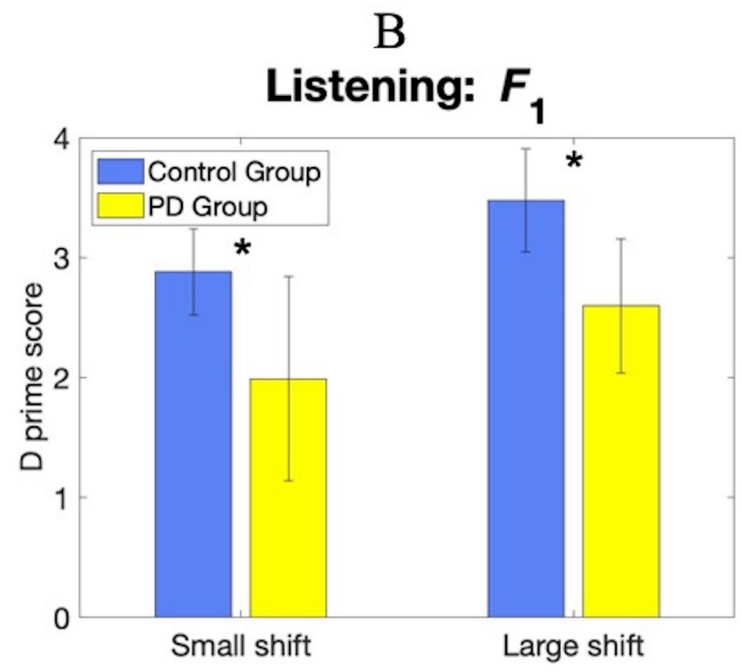

Figure 4.

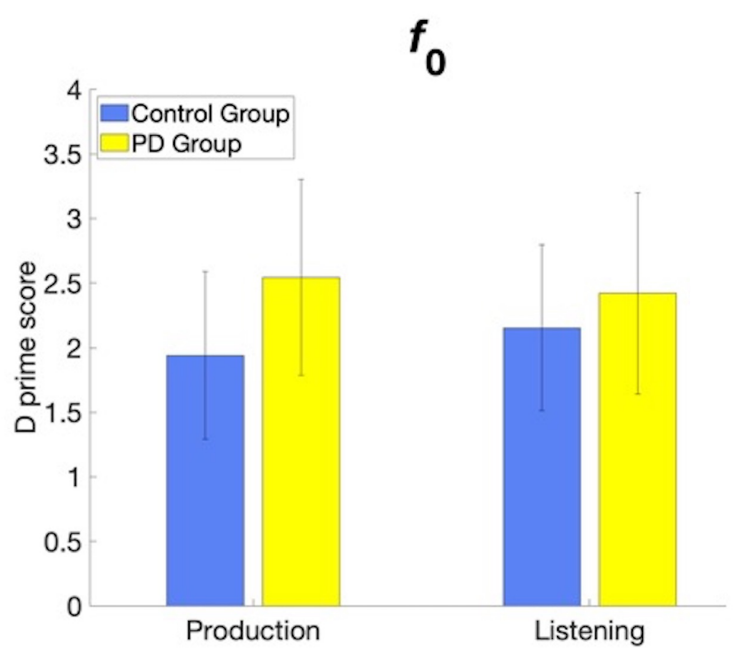

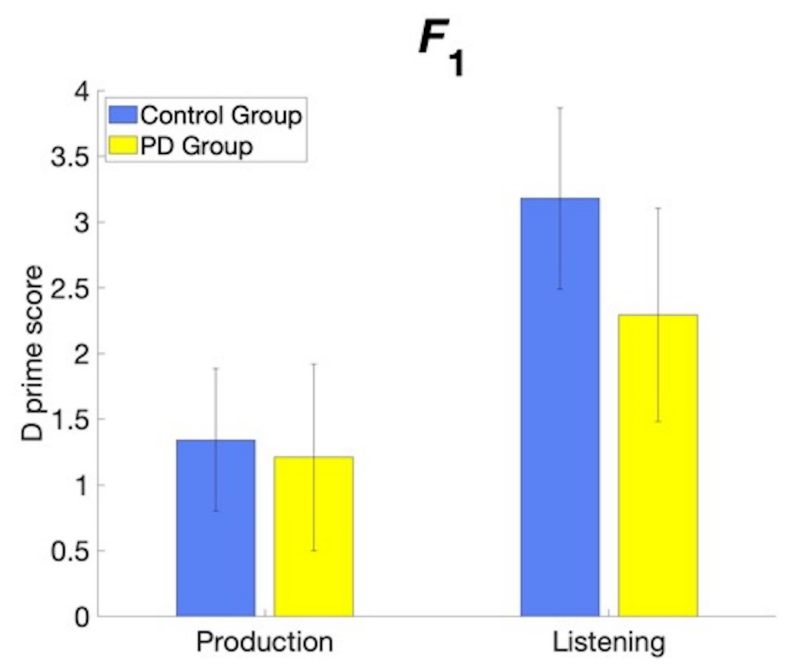


The relationship between speech perceptual discrimination and speech production in Parkinson's disease

Table 1.

Individuals scores of participants with PD on MDS-UPDRS and dysarthria perceptual rating

\begin{tabular}{|c|c|c|c|c|c|c|c|}
\hline & $\begin{array}{l}M D S- \\
U P D R S\end{array}$ & Respiration & Phonation & Resonance & Articulation & Prosody & $\begin{array}{l}\text { Total } \\
\text { speech } \\
\text { score }\end{array}$ \\
\hline PD01 & 15 & 4 & 26 & 3 & 7 & 13 & 53 \\
\hline PD02 & 25 & 4 & 23 & 3 & 7 & 21 & 58 \\
\hline PD03 & 31 & 2 & 16 & 4 & 9 & 15 & 46 \\
\hline PD04 & 24 & 2 & 29 & 3 & 11 & 22 & 67 \\
\hline PD05 & 33 & 3 & 32 & 3 & 11 & 21 & 70 \\
\hline PD06 & 5 & 3 & 22 & 3 & 9 & 13 & 50 \\
\hline PD07 & 23 & 3 & 29 & 3 & 14 & 22 & 71 \\
\hline PD08 & 48 & 3 & 25 & 3 & 13 & 22 & 66 \\
\hline PD09 & 27 & 2 & 23 & 4 & 14 & 16 & 59 \\
\hline PD10 & 13 & 3 & 22 & 3 & 10 & 18 & 56 \\
\hline PD11 & 22 & 3 & 25 & 5 & 12 & 20 & 65 \\
\hline PD12 & 3 & 2 & 16 & 4 & 8 & 15 & 45 \\
\hline PD13 & 22 & 2 & 28 & 3 & 10 & 18 & 61 \\
\hline PD14 & 33 & 3 & 20 & 3 & 10 & 18 & 54 \\
\hline PD15 & 38 & 2 & 16 & 3 & 7 & 14 & 42 \\
\hline
\end{tabular}


The relationship between speech perceptual discrimination and speech production in Parkinson's disease

\section{Table 2.}

Correlational analysis between $f_{\mathrm{o}}, F_{1}$ error detection and MDS-UPDRS, dysarthria perceptual total scores in PD; R Pearson correlation value

\begin{tabular}{llll}
\hline Manipulation & Task & MDS-UPDRS & $\begin{array}{l}\text { Total dysarthria } \\
\text { score }\end{array}$ \\
\hline$f_{0}$ & Production & -0.03 & -0.00 \\
\hline$F_{1}$ & Production & -0.30 & -0.17 \\
\hline$f_{0}$ & Listening & -0.31 & -0.33 \\
\hline$F_{1}$ & Listening & -0.50 & -0.56 \\
\hline
\end{tabular}

